Editorial

\title{
Acknowledgement to Reviewers of Agriculture in 2015
}

\author{
Agriculture Editorial Office
}

Published: 2 February 2016

MDPI AG, Klybeckstrasse 64, CH-4057 Basel, Switzerland; agriculture@mdpi.com

The editors of Agriculture would like to express their sincere gratitude to the following reviewers for assessing manuscripts in 2015.

We greatly appreciate the contribution of expert reviewers, which is crucial to the journal's editorial decision-making process. Several steps have been taken in 2015 to thank and acknowledge reviewers. Good, timely reviews are rewarded with a discount off their next MDPI publication. By creating an account on the submission system, reviewers can access details of their past reviews, see the comments of other reviewers, and download a letter of acknowledgement for their records. In addition, MDPI has launched a collaboration with Publons, a website that seeks to publicly acknowledge reviewers on a per journal basis. This is all done, of course, within the constraints of reviewer confidentiality. Feedback from reviewers shows that most see their task as a voluntary and mostly unseen work in service to the scientific community. We are grateful to our reviewers for the contribution they make.

$\begin{array}{lll}\text { Abedin, Joinal } & \text { Gwynn-Jones, Dylan } & \text { Nastasi, Benedetto } \\ \text { Adégbidi, Hector Guy } & \text { Habteselassie, Mussie } & \text { Neigh, Christopher } \\ \text { Aguirre-Villegas, Horacio Andres } & \text { Halterman, Dennis } & \text { Newman, Yoana } \\ \text { Aiken, Glen E } & \text { Hannaford, Susannah } & \text { Nichols, Shirley } \\ \text { Albrecht, Julie } & \text { Hassan, Md. Kamrul } & \text { Niemira, Brendan A. } \\ \text { Alexopoulos, Athanassios } & \text { He, Zhongqi } & \text { O'Shea, Cormac } \\ \text { Alfonzo, Antonio } & \text { Heiden, Uta } & \text { Osseweijer, Patricia } \\ \text { Alonso, Garcia } & \text { Hennessy, Deirdre } & \text { Pacheco, David } \\ \text { Altieri, Giuseppe } & \text { Henry, Clémence } & \text { Palomo-Ríos, Elena } \\ \text { Anceno, A. J. } & \text { Hestrin, Rachel } & \text { Pascual, Nuria } \\ \text { Anderson, Randy L } & \text { Hill, David } & \text { Patil, Sonal } \\ \text { Ângelo Rodrigues, M } & \text { Hille, Stefanie } & \text { Pau-Roblot, Corinne } \\ \text { Arazuri, Silvia } & \text { Ho, Shin-Lon } & \text { Paul, George } \\ \text { Arumugam, Surendran } & \text { Hoque, Sonia } & \text { Pearson, Simon } \\ \text { Aruoma, Okezie } & \text { Hornung, Andreas } & \text { Pekrun, Carola } \\ \text { Atkinson, Laura D. } & \text { Hristov, Alexander } & \text { Perkins, Lora } \\ \text { Badawy, Amr El } & \text { Huber, Korinna } & \text { Pinotti, Luciano } \\ \text { Bailly, Jean-Denis } & \text { Hwang, Cheng-An } & \text { Pizzeghello, Diego } \\ \text { Ballizany, Wouter } & \text { Ibrahim, Salam A. } & \text { Pliakoni, Eleni } \\ \text { Bampidis, Vasileios } & \text { Ieropoulos, Ioannis } & \text { Posmanik, Roy } \\ \text { Barjolle, Dominique } & \text { Ilmakunnas, Pekka } & \text { Prussin, Aaron J } \\ \text { Barker, David } & \text { Imeson, Anton } & \text { Pryor, Scott W. } \\ & & \end{array}$




\begin{tabular}{|c|}
\hline Barnes, Jessica \\
\hline Belcher, Ken \\
\hline Biasioli, Franco \\
\hline Bolzoni, Giuseppe \\
\hline Bonfil, David \\
\hline Bos-Brouwers, Hilke \\
\hline Boström, Dan \\
\hline Breulmann, Marc \\
\hline Brock, Christopher J. \\
\hline Brown, Robert \\
\hline Burger, Hannah \\
\hline Burud, Ingunn \\
\hline Butrón, Ana \\
\hline Cabrera, Raimundo \\
\hline Calabrò, Serena \\
\hline Campbell, Petya \\
\hline Carlson, Alicain \\
\hline Caslake, Laurie \\
\hline Chang, Matt \\
\hline Chardon, Fabien \\
\hline Cheplick, Gregory \\
\hline Corton, John \\
\hline Costa, Joaquim \\
\hline Creamer, Rebecca \\
\hline Cressey, Peter \\
\hline Cresswell, James E \\
\hline Cutler, Stephen J. \\
\hline De Cesare, Fabrizio \\
\hline De Feo, Vincenzo \\
\hline De Rensis, Fabio \\
\hline De Salvo, Maria \\
\hline Desjardins, Raymond \\
\hline Di Tullo, Pamela \\
\hline Dios-Palomares, Rafaela \\
\hline Djanibekov, Utkur \\
\hline Döring, Thomas \\
\hline Du, Liangcheng \\
\hline Dubeuf, Jean-Paul \\
\hline Ducatelle, Richard \\
\hline Easton, Herrick Sydney \\
\hline El-Wakeil, Nabil \\
\hline Estellés, Fernando \\
\hline Faleiro, Maria L. \\
\hline
\end{tabular}

Inyang, Mandu

Irving, Louis

Jain, Mohan Shri

Jaiswal, Deepak

Jansa, Jan

Jansson, Nicklas

Jarén, Carmen

Jayanty, Sastry

Jha, Rajesh

Jones, David

Kagan, Isabelle A.

Kah, Melanie

Karydas, Christos G.

Kavvadias, Victor

Kawamura, Kensuke

Kitinoja, Lisa

Klopfenstein, Terry

Klotz, James

Knight, Anthony

Kobayashi, Mimako

Krishna, Vijesh V.

Kwapinski, Witold

Lacava, Teodosio

Laird, David

Lamothe, Laurence

Lanza, Massimiliano

Lazarovits, George

Lehmhus, Jörn

Lemaire, Gilles

Lewis, Kathleen Anne

Li, Deying

Li, Hong

Li, Qian

Libra, Judy

Lin, Amy Hui-Mei

Linse, Lisbeth

Liu, Shufan

Lohakare, Jayant

Lopez, Gabriela

Lundin, Lisa

Luo, Meng

Luquet, Delphine

Luth Richter, Jessika
Quinlan, Jennifer

Rai, Dilip K.

Ramírez-Restrepo, Carlos

Ravindran, Ravi

Reed, Stewart

Resop, Jonathan

Rigby, Hannah

Rodilla, Jesus M.

Rogovska, Natalia

Romera, Alvaro

Rosin, Chris

Ruiz-Aceituno, Laura

Safferman, Steve

Salgueiro, Ligia

Sarath, Gautam

Sattar, Anwar

Schaeffer, Robert

Scott, Tom

Sela, Shlomo

Senwo, Zachary

Shan, Xueyan

Shill, Kierston

Sillanpaa, Mika

Silva, Brenner

Sivakumar, Dharini

Skoulou, Vassiliki K.

Spiertz, Huub

Steinberger, Yosef

Stöber, Silke

Suslow, Trevor V.

Szolnoki, Gergely

Takashi, Nishizawa

Talukder, Shyamal Krisna

Tenza-Abril, Antonio José

Tetard-Jones, Catherine

Thomas, Mathieu

Thorne, Mark

Timmermans, Toine

Tits, Laurent

Toivonen, Peter

Tresoldi, Grazyne

Trevisani, Marcello

Tully, Katherine 
Farrell, Robert

Fernández, Belén

Fernández, Camino

Fernandez-Escobar, Ricardo

Filiatrault, Melanie

Fountas, Spyros

Franks, Jeremy Robert

Franz, Chlodwig

Friedli, Michael

Friedrich, Theodor

Galyean, Michael L.

Gan, Serella

Gao, Xiangpeng

Garcia-Sanchez, Antonio-Javier

Gardner, Dale

Gatzia, Dimitria

Gebremedhin, Kifle

Georgiou, Constantinos A.

Gianniantonio, Petruzzelli

Gioannis, Giorgia De

Glenna, Lealand L.

Gonzalez, Emilio

González-Coloma, Azucena

Gross, Jürgen

Gruber, Margie

Gruber, Sabine
Madibela, Othusitse Ricky Udo, Henk

Magrassi, Fabio

Vandervoort, Chris

Mahajan, Devinder

Via, Brian

Mahieu, Maurice

Vittori, Sauro

Mahmud, M. Rizaludin

Maja, Joe Mari

Vlysidis, Anestis

Voigt, Thomas

Voroney, Paul

Voss, Kenneth A.

Mantell, Sinclair

Walton, David

Manue, Nieves-Cordones

Walz, Yvonne

Marín, Sonia

Wang, Jianying

Marques, Maria Jose

Martínez-Mena, María

Wang, Yan

Maurer, John

Webb, Gary W.

Maynard, Jonathan

Wellstein, Camilla

McHenry, Mark P.

Wibowo, Santoso

McManus, Sarah

Williams, Barbara

Mercier, Yves

Willms, Walter D.

Micallef, Shirley

Mills, Aaron

Wreford, Anita

$\mathrm{Xu}$, Chengyuan

Mirosa, Miranda

Yang, Suann

Mobini, Saeid

Yang, Qianru

Moore, Darrell

Zhang, Yifeng

Moral, Raul

Zhang, Qian

Morris, Nathan

Morrissey, Karyn

Zhu, Mingming

Zia, Huma

Mostrom, Michelle S.

(C) 2016 by the author; licensee MDPI, Basel, Switzerland. This article is an open access article distributed under the terms and conditions of the Creative Commons by Attribution (CC-BY) license (http://creativecommons.org/licenses/by/4.0/). 